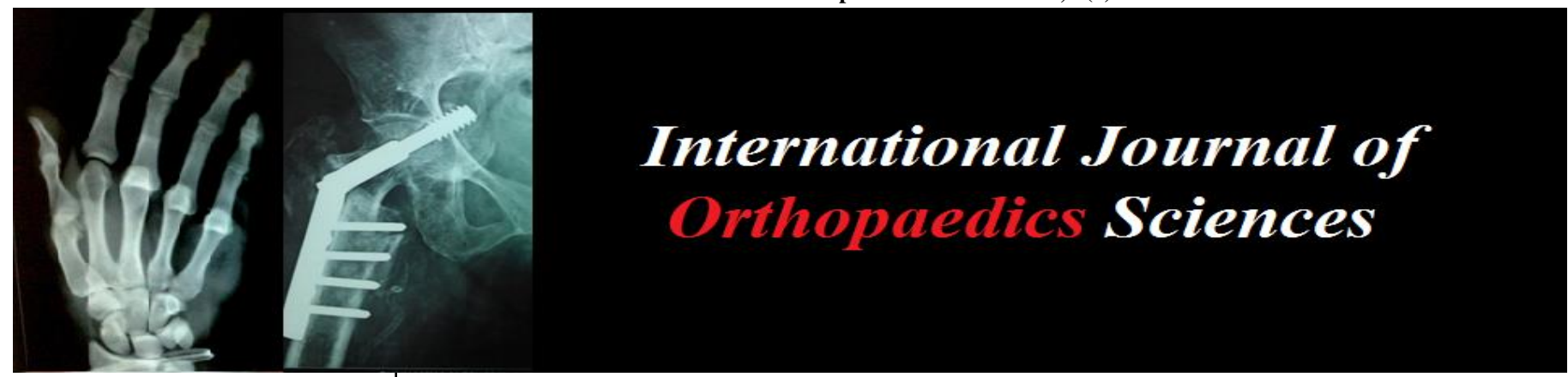

E-ISSN: 2395-1958

P-ISSN: 2706-6630

IJOS 2020; 6(1): 443-445

(C) 2020 IJOS

www.orthopaper.com

Received: 16-11-2019

Accepted: 20-12-2019

Kone Samba

Department of Orthopaedics,

University Hospital Center,

Abidjan, Côte d'Ivoire

Kone Seydou GN

Department of Orthopaedics, University Hospital Center,

Abidjan, Côte d'Ivoire

\section{Godefroie Adibo}

Department of Orthopaedics, University Hospital Center,

Abidjan, Côte d'Ivoire

Agoh Serge

Department of Orthopaedics,

University Hospital Center,

Abidjan, Côte d'Ivoire
Corresponding Author: Kone Samba

Department of Orthopaedics, University Hospital Center,

Abidjan, Côte d'Ivoire

\section{Triple injury association treated by dynamic muscle transfer: Clinical case about acromioclavicular disjunction associated with coracoid fracture and rupture of coraco-clavicular ligaments}

\author{
Koné Samba, Koné Seydou GN, Godefroie Adibo and Agoh Serge
}

DOI: https://doi.org/10.22271/ortho.2020.v6.i1h.1904

\begin{abstract}
Acromioclavicular disjunction is a frequent traumatic lesion, but one that often goes unnoticed. Its association with coracoid process fracture / or a rupture of the coraco-clavicular ligaments is infrequent or even exceptional.

We report the case of a young man polytraumatized by traffic road and who also presented: acromioclavicular disjunction, a coracoid process fracture, and a rupture of the coraco-clavicular ligaments. The surgical treatment carried out was an acromioclavicular fixation by a screwing associated with dynamic muscle transfer (Dewar Barrington technique). After 8 months follow-up the functional result was satisfactory. The aim was to highlight this rare clinical entity and to discuss its therapeutic modalities and prognostic.
\end{abstract}

Keywords: Acromioclavicular, coracoid, coraco-clavicular ligaments, muscle transfer

\section{Introduction}

Acromioclavicular disjunction is a frequent lesion, but one that often goes unnoticed. Its association with the coracoid process fracture is uncommon. It is most often a tear-away fracture of the base of the coracoid which remains attached to the clavicle via the coracoclavicular ligaments which have remained intact) ${ }^{[1]}$. The rupture of these ligaments during this injury association is an exceptional entity) ${ }^{[2]}$. Rigorous and methodical physical examination is essential in order to make an accurate diagnosis, the only guarantor of care and adequate for an optimal functional result.

It's good to have correct conventional radiographs that should lead to the slightest doubt in the prescription of a computed tomography (CT) or magnetic resonance imaging (MRI). The therapeutic management of this triple association injury is not yet unequivocal even if the majority of authors prefer the surgical means.

We report a case of this triple association injury in an adult occurring in polytrauma. The aim was to highlight this rare clinical entity and to discuss its therapeutic modalities and prognostic.

\section{Case report}

It was a 29-year-old mason, admitted to emergency room for polytrauma following a road traffic (motorcyclist struck by a vehicle). The Injury Severity Score was 45. The lesion assessment noted: head trauma with initial loss of consciousness, a posterior arch of the 11th left rib fracture, of a hemoperitoneum table (traumatic rupture of the spleen) and large painful at left shoulder as a whole with relative functional impotence of the left upper limb. The clinical examination noted elective pain at left shoulder, exacerbated by the movements of the shoulder and especially during the antepulsion and the horizontal abduction of the arm. In the outer third of the clavicle there was abnormal mobility on the piano key, an anteroposterior drawer reflecting the existence of an acromioclavicular dislocation.

Conventional radiography of left shoulder revealed acromioclavicular disjunction stage III of 
Rockwood's) [3]. In front of the abdominal emergency, a simple waiting immobilization of the shoulder by an elbowto-body scarf was performed. The hemoperitoneum was treated urgently by laparotomy (splenectomy). A left shoulder CT scan was requested, but the patient could not honor it. On the 10th post-laparotomy day (after healing), surgical management of the acromioclavicular disjunction was undertaken. Surgical approach started from the delto-pectoral groove, joining the collarbone and became horizontal until the acromioclavicular joint. (Figure 1).

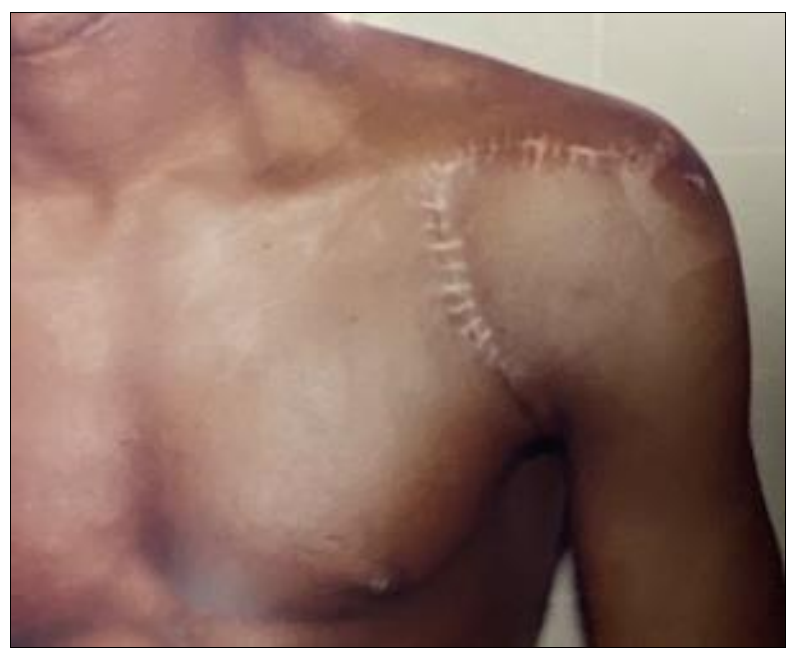

Fig 1: Local appearance of left shoulder, surgical approach.

The lesion assessment was an intraoperative surprise: an acromioclavicular disjunction, a fracture-tearing of the base of the coracoid process, a rupture of the coraco-clavicular ligaments, but an integrity of the delto-pectoral chappe.

First, the acromioclavicular joint was screwed on. Given the impossibility of carrying out a solid osteosynthesis of the coracoid process and of correctly repairing the dilated coracoclavicular ligaments, we have associated with this screwing a dynamic muscle transfer (ligamentoplasty) according to Dewar and Barrington technique) ${ }^{[4]}$. It consists of a transfer of the tip of the coracoid on which the insertions of the pectoralis and the coraco-brachial are preserved. The point of the coracoid is fixed to the anterior part of the collarbone by a screw. A shoulder scarf was put on for 6 weeks. Postoperative radio occurred satisfactory restoration of acromioclavicular and coraco-clavicular joint. (Figure 2)

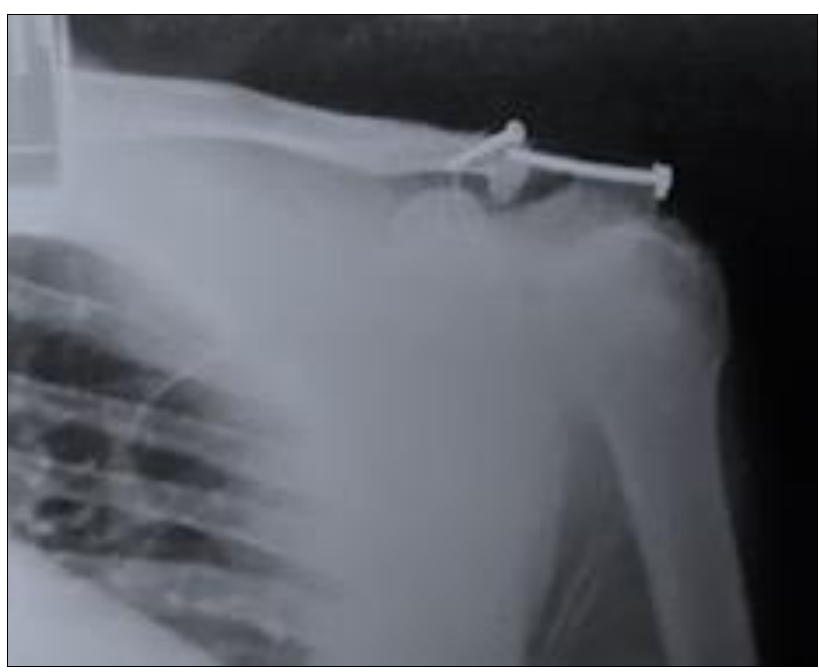

Fig 2: Shoulder X-ray (A) Antero-posterior; showing screws, and restoration of acromioclavicular and coraco-clavicular joint
The post-operative follow-ups were simple with rehabilitation from the 5 th post-operative.

After 8 months the clinical evaluation according, Constant score was 94\% (Pain 14/15 - Activity level 18/20 - Mobility 38/40 - Strength 24/25). Radiographically there were no abnormal signs (of osteoarthritis, non-union, calcification). Resumption of socio-professional activities was possible from the 5th month.

\section{Discussion}

The purpose of postponing this unusual clinical case in a young adult in the aftermath of multiple trauma was to discuss the therapeutic modalities and prognoses. Fractures of the coracoid process, are frequently associated with acromioclavicular disjunction) ${ }^{[2]}$. It is generally a fall on the stump of the shoulder, with abrupt lowering of the scapula compared to clavicle and forced adduction movement, causing acromioclavicular disjunction with coracoid base fracture, instead of the rupture of the coraco-clavicular ligaments which remain intact. The existence of a rupture of these ligamentous formations constitutes an exceptional triple injury association: acromioclavicular disjunction, rupture of the coraco-clavicular ligaments and the base of the coracoid process fracture. Only 3 isolated cases have been described in the literature, the most recent of which is reported by Wang ${ }^{[5]}$ in 1995. No case of this triple injury association has been observed in our department in 20 years of current practice. Our clinical case is distinguished by its circumstance of occurrence (accident on the public highway), its intraoperative diagnosis and by the operating technique used. Unlike our clinical case (road accident), the etiology is dominated by sports accidents (football) in the occurrence of the triple lesion association. These lesions generally affect adolescents and young adults with a clear male predominance. The clinical picture is very expressive but, can go unnoticed because the diagnosis is often difficult. Only the pain caused, and the abnormal mobility on the piano key, draws attention. Conventional radiography makes it possible to diagnose a coracoid process fracture and acromioclavicular disjunction. However, unlike the classic association where the intercoraco-clavicular space is preserved, in case of rupture of the coraco-clavicular ligaments the displacement is greater and the space enlarged. It is of great interest to have correct conventional radiographic incidences which should lead to the slightest doubt in the prescription of a computed tomography (CT) or magnetic resonance imaging (MRI). Regarding the therapeutic attitude, opinions are probably divided because of the rarity of these injury. Majority of authors opt for surgical treatment. The approach (anterior, superior) remains dependent on the surgical procedure to be performed.

For acromioclavicular stabilization, various osteosynthesis processes (pinning-guying, screwing according to Bosworth, coraco-clavicular lacing, and ligamentoplasty) exist. While not all of them have been studied, each has advantages and disadvantages. The choice remains a school affair. Concerning the coracoid process fracture, a simple axial screwing is considered dangerous and difficult by Saragaglia ${ }^{2]}$; it should be associated with stabilization of the acromioclavicular by a shroud with refection of careful treatment of the delto-trapezian clevis. For our part, we agree with Saragaglia) ${ }^{[2]}$ that the existence of a rupture of the coracoclavicular ligaments requires surgical repair either of the process, or of the ligaments, or even of the two lesions. The theory that the coracoid is self-stable is not valid.

Faced with the impossibility of carrying out a solid 
osteosynthesis of the coracoid process and correctly repairing the dilated coraco-clavicular ligaments, we have associated a ligamentoplasty by a dynamic muscular transfer (technique of Dewar Barrington) ${ }^{[4]}$. Which consists in transposing by screwing the tip of the pedunculated coracoid process to the coracid biceps at the level of the lower face of the collarbone. Currently, there is no benchmark score or classification to assess the results of surgical repair of this triple injury association due to lack of mass production. Functionally, our patient was very satisfied (Constant score at 94\%) to have obtained his autonomy and resumed his socio-professional activities.

\section{Conclusion}

The association of acromioclavicular disjunctions, coracoid process fracture and a rupture of the coraco-clavicular ligaments is exceptional.

Difficult diagnosis, an anamnesis and rigorous clinical examination associated with radiological investigations (conventional, CT, MRI) of quality will allow a precise lesion assessment. The treatment of choice is surgical, aimed at stabilizing the acromioclavicular joint and fixing the coracoid process and / or repairing the ruptured ligaments.

For our part we recommend the technique of Dewar Barrington, which gave us full satisfaction.

Conflict of interests: The authors declare no conflict of interest.

\section{Author's contributions}

All the authors contributed to the writing of this manuscript, had read and approved the final version.

\section{References}

1. Moison J, Battikha M, Madi A. Fracture arrachement de l'apophyse coracoïde associée à une luxation acromioclaviculaire. Journal de traumatologie du sport. 1992; 9(3):173-177.

2. Saragaglia D et al. Disjonction acromio-claviculaire et fracture de la base du processus coracoïde: A propos de 2 cas. Journal de Traumatologie du sport. 1993; 10.3:179184.

3. Rockwood CA. Injuries to the acromioclavicular joint. In: Rockwood CA, Grenn DP, eds. Fractures in adults, 2nd ed. Philadelphia: Lippincott. 1984; 1:860.

4. Dewar FP, Barrington TW. The treatment of chronic Acromio-Clavicular dislocation. J Bone Joint Surg Br. 1965; 47:32-5.

5. Wang KC, Hsu KY, Shih $\mathrm{CH}$. Coracoid process fracture combined with acromioclavicular dislocation and coracoclavicular ligament rupture. A case report and review of the literature. Clinical orthopaedics and related research. 1994; 300:120-122. 